\title{
Waldenstrom macroglobulinemia - indolent disorder or slow killer?
}

Authors: Iva Ljubas ${ }^{1}$, Ozana Miličević ${ }^{1}$, Inga Mandac Smoljanović ${ }^{2}$ (mentor)

${ }^{1}$ School of Medicine, University of Zagreb, Zagreb, Croatia

${ }^{2}$ Clinical Hospital Merkur, Department of Hematology, Zagreb, Croatia

DOI: https://doi.org/10.26800/LV-142-supp5-19

\section{Background:}

Waldenström macroglobulinemia (WM) is an indolent (slow-growing) subtype of non-Hodgkin lymphoma that affects small lymphocytes. The risk of WM increases with age with median age at diagnosis of 63 years. Men are more likely than women to develop the WM. The overall incidence of WM is approximately five cases per one million persons per year

\section{Case presentation:}

Many patients have constitutional symptoms (fevers, night sweats, weight loss), but some patients can be asymptomatic even for several years. WM is characterized by over production of monoclonal IgM and in some cases increased IgM can result in hyperviscosity syndrome (HVS). HVS refers to an increase in serum viscosity secondary to circulating proteins and results in a variety of symptoms like visual symptoms, altered mental status, stroke or congestive heart failure. The diagnosis is made by clinical and laboratory findings, like increased serum protein levels and increased serum viscosity. The patient should receive prompt therapy to avoid progression to multisystem organ failure and prevent life-threatening complications. We report a case of a 51-year-old man with a 4-year history of increased IgM and untreated WM who presented with HVS. His plasma viscosity was $10.94 \mathrm{mPa}$.s. He underwent diagnostic workup and was finally diagnosed with WM ISS 1.

\section{Conclusion:}

Even though Waldenstrom macroglobulinemia is a rare disease and can be asymptomatic for several years, and can present with a variety of symptoms, it should always be considered as a possible differential diagnosis in patients with increased IgM and treated properly.

Keywords: lymphoma, hyperviscosity syndrome, macroglobulinemia 